\title{
Edge Disjoint Hamiltonian Cycles in Eisenstein-Jacobi Networks
}

\author{
Zaid A. Hussain ${ }^{\mathrm{a}}$, Bella Bose ${ }^{\mathrm{b}}$, Abdullah Al-Dhelaan ${ }^{\mathrm{c}}$ \\ ${ }^{a}$ Computer Science Department, College of Computing Sciences and Engineering, \\ Kuwait University, P.O. Box 5969, Safat, Kuwait 13060.E-mail: zhussain@cs.ku.edu.kw \\ ${ }^{b}$ School of Electrical Engineering and Computer Science, Oregon State University, 1148 \\ KEC, Corvallis, OR 97331-3902. E-mail: bose@eecs.orst.edu \\ ${ }^{c}$ Department of Computer Science, College of Computer and Information Sciences, King \\ Saud University, P.O. Box 51178, Riyadh 11543, King Saudi Arabia. E-mail: \\ dhelaan@ksu.edu.sa
}

\begin{abstract}
Many communication algorithms in parallel systems can be efficiently solved by obtaining edge disjoint Hamiltonian cycles in the interconnection topology of the network. The Eisentein-Jacobi (EJ) network generated by $\alpha=a+b \rho$, where $\rho=(1+i \sqrt{3}) / 2$, is a degree six symmetric interconnection network. The hexagonal network is a special case of the EJ network that can be obtained by $\alpha=a+(a+1) \rho$. Generating three edge disjoint Hamiltonian cycles in the EJ network with generator $\alpha=a+b \rho$ for $\operatorname{gcd}(a, b)=1$ has been shown before. However, this problem has not been solved when $\operatorname{gcd}(a, b)=d>1$. In this paper, some results to this problem are given.
\end{abstract}

Keywords: Parallel computing; Eisenstein-Jacobi network; Edge disjoint, Hamiltonian cycle; interconnection networks.

\section{Introduction}

Interconnection topology plays a major role in achieving high performance in parallel systems. In the past, many interconnection topologies have been proposed - some most popular being hypercube, torus, mesh, De Bruijn networks, etc. [10][8]. Recently, an efficient interconnection topology called EJ network has been proposed in [15] and some further properties of this network have been studied in [2][9]. Furthermore, the hexagonal network is reviewed in Section 2.

Preprint submitted to Journal of Parallel and Distributed Computing

June 21, 2015

(C) 2015. This manuscript version is made available under the Elsevier user license http://www.elsevier.com/open-access/userlicense/1.0/ 
The Hamiltonian decomposition of a given network is one of the important topological properties, where a Hamiltonian cycle in a network graph is a cycle containing all the nodes in the graph $[10][7][8]$. In the case of edge disjoint decomposition, we need to find the maximum number of edge disjoint Hamiltonian cycles of the given network. Some of the applications of the Hamiltonian decomposition of a given network are briefly described below.

1. Consider the all-to-all communication algorithm, where each node sends a message to all other nodes. Assume the number of nodes in the network is $N$. In a single I/O port model, where each node can send and receive a single message at a time, an optimal algorithm can be obtained as follows. First form a Hamiltonian cycle in the network and then in the $i$-th step, $i=1,2,3, \ldots, N-1$, each node can send the message which it has been received from the previous node in the $(i-1)$-th step to the next node by keeping a copy of it to itself. This algorithm takes $N-1$ steps. Since a node can receive at most one message at a time and it has to receive $N-1$ messages, this algorithm is optimal. Now, suppose each node has degree $d$ and this system can support multi-port $\mathrm{I} / \mathrm{O}$, meaning each node can send and receive $d$ messages in unit time. In this case, if we are able to construct $d / 2$ edge disjoint Hamiltonian cycles then each message of size $m$ can be partitioned into messages of size $(2 m) / d$ and each of these can be sent along $d / 2$ edge disjoint Hamiltonian cycles. Thus, the time complexity of the algorithm can be improved by a factor of $d / 2$.

2. Edge disjoint cycles are useful in the case of fault tolerant routing. If the network has $d$ edge disjoint Hamiltonian cycles then even in the presence of $2 d-1$ edge failures a node can communicate with any other node.

3. The property of two edge disjoint Hamiltonian cycles in Gaussian networks is used to develop bufferless routing in optical Gaussian microchip interconnect [7].

Edge disjoint Hamiltonian cycles have been studied for varies networks in the past [3][4][5][6][12][13][14][16][17]. For EJ network with generator $\alpha=$ $a+b \rho, \operatorname{gcd}(a, b)=1$ a method to generate three edge disjoint Hamiltonian cycles is given in [9]. However, when $\operatorname{gcd}(a, b)=d>1$, this problem has not been solved till now. In this paper, we develop a method to generate three 
edge disjoint Hamiltonian cycles for EJ network generated by $\alpha=a+b \rho$, even when $\operatorname{gcd}(a, b)=d \geq 1$. Since the degree of each node in this network is 6 the maximum number of edge disjoint Hamiltonian cycles that can be obtained in these networks is 3 and the solution is optimal.

The main ideas of the paper are as follows. First, for a given EJ network generated by $\alpha=a+b \rho, \operatorname{gcd}(a, b)=d>1$, a rectangular representation with $d$ rows and $r=\left(a^{2}+b^{2}+a b\right) / d$ columns is given. Then, it is shown that there exist three disjoint sets of $d$ cycles each of length $r$, the first set of $d$ cycles along the horizontal dimension, the second set along the vertical dimension, and the third set along the diagonal dimension. By exchanging some proper sets of horizontal and vertical edges, the horizontal cycles and vertical cycles are connected to form two edge disjoint Hamiltonian cycles. Then, some proper set of edges in the modified first Hamiltonian cycle is exchanged with the same number of proper diagonal edges to form two edge disjoint Hamiltonian cycles. Thus, we get three edge disjoint Hamiltonian cycles.

The rest of the paper is structured as follows. In Section 2, we briefly review some topological properties of EJ networks. The previous work on edge disjoint Hamiltonian cycle in EJ network generated by $\alpha=a+b \rho$ with $\operatorname{gcd}(a, b)=1$ is reviewed in Section 3. In Section 4, we introduce the rectangular representation of EJ networks. This representation is useful in obtaining the main results in Section 5. Section 5 describes the solution of finding three edge disjoint Hamiltonian cycles in EJ networks when $\operatorname{gcd}(a, b)=d>1$. Finally, the paper is concluded in Section 6 .

\section{Eisenstein-Jacobi Networks}

\subsection{EJ Integers}

The EJ networks are designed based on the concept of EJ integers, which we defined below. The Eisenstein-Jacobi integer $\mathbb{Z}[\rho]$ is defined as

$$
\mathbb{Z}[\rho]=\{x+y \rho \mid x, y \in \mathbb{Z}\}
$$

where $\rho=(1+i \sqrt{3}) / 2$, and $i=\sqrt{-1}$. It is known that $\mathbb{Z}[\rho]$ is a Euclidean domain and the norm of EJ integer $\alpha=a+b \rho$ is given by $N(\alpha)=a^{2}+b^{2}+$ $a b$. The norm means, under residue class modulo (i.e., under the possible remainders) $\alpha=a+b \rho$ there will have exactly $a^{2}+b^{2}+a b$ distinct EJ integers. More on EJ integers can be seen in [11]. Note that it can be seen that $\rho^{2}=\rho-1, \rho^{3}=-1, \rho^{4}=-\rho, \rho^{5}=1-\rho$, and $\rho^{6}=1$. 


\subsection{Definition and Topological Properties of EJ Networks}

EJ networks are based on quotient rings of EJ integers. They are regular symmetric networks of degree six, that is each node has six neighbors. The total number of nodes in the network is $N(\alpha)=a^{2}+b^{2}+a b$. Each node in the network is labeled by $x+y \rho$. Two nodes $A$ and $B$ are said to be adjacent if and only if $(A-B) \bmod \alpha$ is equal to $\pm 1, \pm \rho$, or $\pm \rho^{2}$. The formal definition is described below.

Definition 1 ([15]). Given $0 \neq \alpha=a+b \rho \in \mathbb{Z}[\rho]$ where $\rho=(1+i \sqrt{3}) / 2$ and $0 \leq a \leq b$, the Eisenstein-Jacobi network is defined as a graph $E J_{\alpha}(V, E)$ where:

1. $V=\mathbb{Z}[\rho]_{\alpha}=\{x+y \rho \bmod \alpha \mid x, y \in \mathbb{Z}\}$ is the node set.

2. $E=\left\{(\beta, \gamma) \in V \times V \mid(\beta-\gamma) \equiv \pm 1, \pm \rho, \pm \rho^{2} \bmod \alpha\right\}$ is the edge set.

We refer $E J_{\alpha}$ as the Eisenstein-Jacobi network generated by $\alpha$. The following theorem describes the distance distribution of the network, which is the number of nodes at distance $s$, for $s=0,1, \ldots, M$, where $M=(a+2 b) / 3$. This number is denoted as $W T_{E J}(s)$.

Theorem $1([9])$. Let $0 \neq \alpha=a+b \rho \in \mathbb{Z}[\rho]$ such that $0 \leq a \leq b, T=$ $(a+b) / 2, M=(a+2 b) / 3$. The diameter of this network is at most $M$ and the distance distribution of the network $E J_{\alpha}$ is as follows:

$$
W T_{E J}(s)= \begin{cases}1 & \text { if } s=0 \\ 6 s & \text { if } 1 \leq s<T \\ 18(M-s) & \text { if } T<s<M \\ 2 & \text { if } b \equiv a(\bmod 3) \text { and } s=M \\ 0 & \text { if } s>M \\ N(\alpha)-\sum_{s=0, s \neq T}^{M} W T_{E J}(s) & \text { if } s=T\end{cases}
$$

Example 1. Figure 1 illustrates the node distribution of EJ network generated with $\alpha=3+4 \rho$ where the center node, i.e. node 0 , is the black node. 


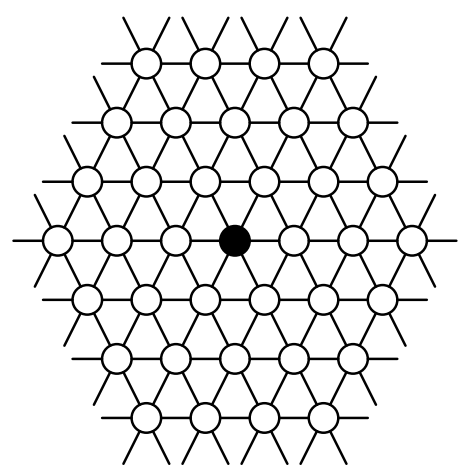

Figure 1: EJ Network Generated with $\alpha=3+4 \rho$.

\section{Previous Work}

Given an EJ network generated with $\alpha=a+b \rho$ such that $0 \leq a \leq b$ and $\operatorname{gcd}(a, b)=1$, the EJ network can be partitioned into three edge disjoint Hamiltonian cycles as follows [9].

For $j=1, \rho$, or $\rho^{2}$, start at node 0 and traverse the path by adding $j \bmod \alpha$. Since the path has a limited number of nodes, at some point, a node, say node $\beta$, will repeat. i.e., there exists a node $\beta$ and an integer $k>0$ such that $\beta+k j \equiv \beta(\bmod \alpha)$. i.e., $k . j \equiv 0(\bmod \alpha)$. Here, we only show that for $j=1, k=|\alpha|^{2}=N(\alpha)$ and hence the cycle is Hamiltonian. Similar proofs can be given when $j=\rho$ and $j=\rho^{2}$.

Since $k \equiv 0(\bmod \alpha), \exists x+y \rho \in \mathbb{Z}[\rho]$ such that

$$
\begin{aligned}
k & =(x+y \rho)(a+b \rho) \\
& =a x+(b x+a y) \rho+b y \rho^{2}
\end{aligned}
$$

Since $\rho^{2}=\rho-1,(2)$ gives

$$
\begin{aligned}
k & =(a x-b y)+(b x+a y+b y) \rho \\
& =(a x-b y)+(a y+b(x+y)) \rho
\end{aligned}
$$

Since $k$ is an integer, $a y+b(x+y)=0$ with $\operatorname{gcd}(a, a+b)=\operatorname{gcd}(a, b)=1$. Therefore, there exists an integer $c \geq 1$ such that $(x, y)=c(a+b,-b)$. Thus

$$
\begin{aligned}
k & =a x-b y=c a(a+b)+c b^{2} \\
& =c\left(a^{2}+b^{2}+a b\right) \\
& \geq a^{2}+b^{2}+a b=|\alpha|^{2}
\end{aligned}
$$


Moreover, the cycles are edge disjoint since the edges used in the first, second, and the third cycles are respectively along the $\rho^{0}=1, \rho$, and $\rho^{2}$ dimensions. This proves the cycle is Hamiltonian.

Example 2. Based on Example 1, Figure 2 shows the first, second, and third edge disjoint Hamiltonian cycles in the EJ network generated with $\alpha=3+4 \rho$ and these are respectively obtained by adding successively $1 \bmod \alpha, \rho \bmod \alpha$, and $\rho^{2} \bmod \alpha$ to node 0 .

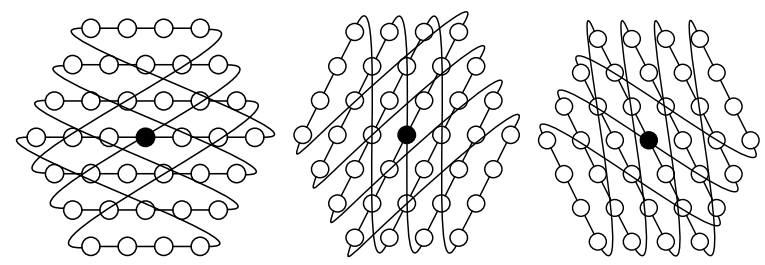

Figure 2: Three Edge Disjoint Hamiltonian Cycles in EJ Network Generated with $\alpha=$ $3+4 \rho$.

\section{Rectangle Representation}

A new representation, called the rectangular representation, of the EJ network generated by $\alpha=a+b \rho$, where $\operatorname{gcd}(a, b)=d>1$ is described in this section. This representation is useful in finding three edge disjoint Hamiltonian cycles.

The idea of this representation is to arrange $a^{2}+b^{2}+a b$ nodes in a rectangle of size $d \times \frac{a^{2}+b^{2}+a b}{d}$, where $d=\operatorname{gcd}(a, b)>1$. The node zero is located at the lower left corner of the rectangle. Each internal node is connected to six other nodes, called neighbors. The nodes at the boundary have wrap-around links with other boundary nodes. The following lemmas and theorem describe the rectangular representation.

Lemma 1. Let $\alpha=a+b \rho, a=a_{1} d, b=b_{1} d, \operatorname{gcd}\left(a_{1}, b_{1}\right)=1$, and $r=$ $\left(a^{2}+b^{2}+a b\right) / d$. Then the numbers $i \rho^{2}+k$ such that $0 \leq i \leq d-1$ and $0 \leq k \leq r-1$ give a complete residue class mod $\alpha$.

Proof. Suppose $\left(i_{1} \rho^{2}+k_{1}\right) \equiv\left(i_{2} \rho^{2}+k_{2}\right) \bmod \alpha$. Then

$$
\begin{aligned}
\rho^{2}\left(i_{1}-i_{2}\right)+\left(k_{1}-k_{2}\right) & =(x+y \rho)(a+b \rho)=a x+(a y+b x) \rho+b y \rho^{2} \\
& =(a x+a y+b x)+(a y+b x+b y) \rho^{2} \\
& =d\left(x\left(a_{1}+b_{1}\right)+a_{1} y\right)+d\left(b_{1} x+\left(a_{1}+b_{1}\right) y\right) \rho^{2}
\end{aligned}
$$


Thus, $i_{1}-i_{2}=d\left[b_{1} x+\left(a_{1}+b_{1}\right) y\right]$. This implies $d \mid\left(i_{1}-i_{2}\right)$. Since $\left|i_{1}-i_{2}\right|<d$

$$
i_{1}-i_{2}=0
$$

Thus, we get

$$
\begin{aligned}
b_{1} x+\left(a_{1}+b_{1}\right) y & =0 \\
\Rightarrow y & =-c b_{1} \\
\Rightarrow x & =c\left(a_{1}+b_{1}\right)
\end{aligned}
$$

And

$$
\begin{aligned}
k_{1}-k_{2} & =c d\left(a_{1}^{2}+b_{1}^{2}+2 a_{1} b_{1}-a_{1} b_{1}\right) \\
& =c d\left(a_{1}^{2}+b_{1}^{2}+a_{1} b_{1}\right) \\
& =c r
\end{aligned}
$$

Since $\left|k_{1}-k_{2}\right|<r$, we get $k_{1}=k_{2}$. Thus, $i \rho^{2}+k, 0 \leq i \leq d-1,0 \leq k \leq r-1$, represent all the elements under $\bmod \alpha$.

The above lemma shows that the nodes in an EJ network can be arranged in a rectangular format of size $d$ rows and $r=d\left(a_{1}^{2}+b_{1}^{2}+a_{1} b_{1}\right)$ columns as shown in Figure 3.

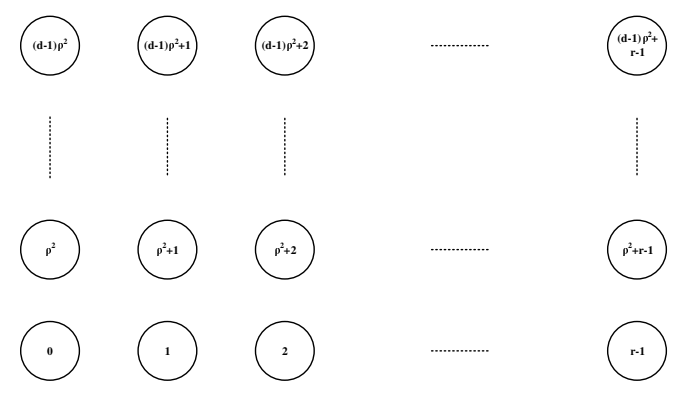

Figure 3: Rectangular Representation of an EJ Network.

A node at the $i$-th row and $j$-th column is given the address $i \rho^{2}+j$, where $0 \leq i<d$ and $0 \leq j<r$. Thus, an internal node $i \rho^{2}+j$ is connected to $i \rho^{2}+j \pm 1, i \rho^{2}+j \pm \rho^{2}$, and $i \rho^{2}+j \pm \rho=i \rho^{2}+j \pm\left(\rho^{2}+1\right)$ as shown in Figure 4.

Now, we need to find out how the boundary nodes are connected. For this, the following lemma is useful. 


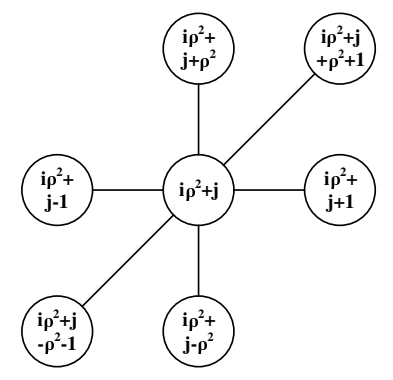

Figure 4: Neighbors of Node $i \rho^{2}+j$.

Lemma 2. Let $r=d\left(a_{1}^{2}+b_{1}^{2}+a_{1} b_{1}\right)$ with $\operatorname{gcd}(a, b)=d, a=a_{1} d$, and $b=b_{1} d$. Then $r \equiv 0 \bmod \alpha$ where $\alpha=a+b \rho$.

Proof. Suppose $r \equiv\left(p+q \rho^{2}\right) \bmod \alpha$, where $0 \leq p<r$ and $0 \leq q<d$. Then

$$
\begin{aligned}
r-\left(p+q \rho^{2}\right) & =(x+y \rho)(a+b \rho) \\
(r-p)-q \rho^{2} & =d\left[a_{1} x+\left(a_{1} y+b_{1} x\right) \rho+b_{1} y \rho^{2}\right] \\
& =d\left[\left(x\left(a_{1}+b_{1}\right)+a_{1} y\right)+\left(b_{1} x+\left(a_{1}+b_{1}\right) y\right) \rho^{2}\right]
\end{aligned}
$$

This implies $d\left(b_{1} x+\left(a_{1}+b_{1}\right) y\right)=-q$ and so $d \mid q$. Since $0 \leq q<d$, we get $q=0$. Therefore $b_{1} x+\left(a_{1}+b_{1}\right) y=0$ and this implies $x=c\left(a_{1}+b_{1}\right)$ and $y=-c b_{1}$ for some integer $c \geq 0$. Thus,

$$
\begin{aligned}
r-p & =d\left(x\left(a_{1}+b_{1}\right)+a_{1} y\right) \\
& =d\left(c\left(a_{1}+b_{1}\right)\left(a_{1}+b_{1}\right)+a_{1}\left(-c b_{1}\right)\right) \\
& =c d\left(a_{1}^{2}+b_{1}^{2}+a_{1} b_{1}\right)=c r
\end{aligned}
$$

Since $0 \leq p<r$ and $c \geq 1$ we get $p=0$. This implies $r \equiv 0 \bmod \alpha$.

The above lemma implies that the boundary node $i \rho^{2}+r-1,0 \leq i<d$, is connected to the node $\left(i \rho^{2}+r\right) \bmod \alpha \equiv i \rho^{2} \bmod \alpha$ as its +1 edge. In addition, the $\rho$-edge of the node $i \rho^{2}+r-1$ for $0 \leq i \leq d-2$ is connected to $\left(i \rho^{2}+r-1\right)+\rho=i \rho^{2}+r-1+\rho^{2}+1=(i+1) \rho^{2}+r \equiv\left((i+1) \rho^{2}\right) \bmod \alpha$. These edges are shown in Figure 5.

Now, we need to show how the $-\rho^{2}$ edges from node $k, 0 \leq k<r$, are connected. For this, we need to find $-\rho^{2}(\bmod \alpha)$. Let $-\rho^{2} \equiv(p+$ 


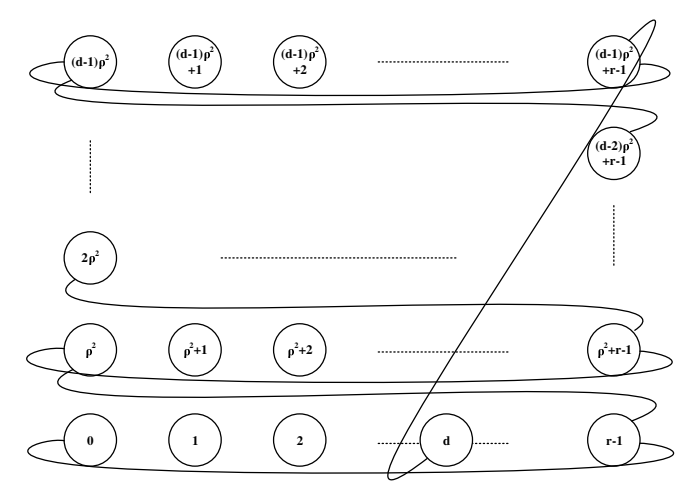

Figure 5: Wrap-around Links of Node $i \rho^{2}+r-1$ for $0 \leq i<d$.

$\left.q \rho^{2}\right) \bmod (a+b \rho)$ where $0 \leq p<r$ and $0 \leq q<d$. Then

$$
\begin{aligned}
-\rho^{2}-p-q \rho^{2} & \equiv 0 \bmod \alpha \\
-p-(1+q) \rho^{2} & =(x+y \rho)(a+b \rho) \\
& =d\left[a_{1} x+\left(a_{1} y+b_{1} x\right) \rho+b_{1} y \rho^{2}\right] \\
& =d\left[\left(a_{1} x+a_{1} y+b_{1} x\right)+\left(a_{1} y+b_{1} x+b_{1} y\right) \rho^{2}\right] \\
& =d\left[\left(a_{1}(x+y)+b_{1} x\right)+\left(a_{1} y+b_{1}(x+y)\right) \rho^{2}\right]
\end{aligned}
$$

This implies $-(1+q)=d\left[a_{1} y+b_{1}(x+y)\right]$. Since $d \mid(-(1+q))$ and $0 \leq q \leq d-1$, we get $q=d-1$. Thus

$$
\begin{aligned}
-(1+d-1) & =d\left[a_{1} y+b_{1}(x+y)\right] \\
1 & =-\left(a_{1} y+b_{1}(x+y)\right)
\end{aligned}
$$

Since $\operatorname{gcd}\left(a_{1}, b_{1}\right)=1$, using the extended GCD algorithm we get

$$
\begin{aligned}
a_{1} A+b_{1} B & =1 \\
\Rightarrow A & =-y \Rightarrow y=-A \\
\Rightarrow B & =-x-y \Rightarrow x=A-B
\end{aligned}
$$

Equating the $\rho^{0}=1$ term in $(3)$, we get

$$
\begin{aligned}
-p & =d\left[a_{1}(x+y)+b_{1} x\right] \\
p & =-d\left[-a_{1} B+b_{1}(A-B)\right]=d\left[a_{1} B+b_{1}(B-A)\right]
\end{aligned}
$$

Note that $\left(A+i b_{1}\right)$ and $\left(B-i a_{1}\right)$ also satisfy the equations (4) and (5). This is because

$$
a_{1}\left(A+i b_{1}\right)+b_{1}\left(B-i a_{1}\right)=a_{1} A+b_{1} B=1
$$


And

$$
\begin{aligned}
p & =d\left[a_{1}\left(B-i a_{1}\right)+b_{1}\left(B-i a_{1}-A-i b_{1}\right)\right] \\
& =d\left[\left(a_{1} B+b_{1}(B-A)\right)-i\left(a_{1}^{2}+b_{1}^{2}+a_{1} b_{1}\right)\right] \\
& =d\left(a_{1} B+b_{1}(B-A)\right)-i d\left(a_{1}^{2}+b_{1}^{2}+a_{1} b_{1}\right) \\
& =d\left(a_{1} B+b_{1}(B-A)\right)-i r \\
& \equiv d\left(a_{1} B+b_{1}(B-A)\right) \bmod r \text { where } 0 \leq p<r .
\end{aligned}
$$

The above argument shows that the $-\rho^{2}$ edge from node $k=0$ is connected to $p+q \rho^{2}$ where $q=d-1$ (i.e., row $d-1$ ) and $p=d\left[a_{1} B+b_{1}(B-A)\right]$, i.e., we need to choose $A$ and $B$ such that $0 \leq d\left[a_{1} B+b_{1}(B-A)\right]<r$. In general, the $-\rho^{2}$ edge from node $k+j, 0 \leq j<r$, is connected to the node $\left((d-1) \rho^{2}+d\left[a_{1} B+b_{1}(B-A)\right]+j\right) \bmod r$.

Similarly, the $-\left(\rho^{2}+1\right)=-\rho$ edge from node $k+j$ is connected to $\left((d-1) \rho^{2}+d\left[a_{1} B+b_{1}(B-A)\right]+j-1\right) \bmod r$.

In summary, based on (5), let $L \equiv p \bmod r$, then the position of node $-\rho^{2}$ in the rectangle representation is $(d-1) \rho^{2}+L$. Now, let $C=x+y \rho^{2}$ be one of the boundary nodes. Then the wrap-around links are defined as:

1. If $0 \leq x<r-L$ and $y=0$ then $C$ is connected to $(x+L)+(d-1) \rho^{2}$ as its $-\rho^{2}$ edge and to $(x+L-1)+(d-1) \rho^{2}$ as its $-\rho$ edge.

2. If $r-L \leq x \leq r-1$ and $y=0$ then $C$ is connected to $(x-r+L)+(d-1) \rho^{2}$ as its $-\rho^{2}$ edge and to $((x-r+L-1) \bmod r)+(d-1) \rho^{2}$ as its $-\rho$ edge.

3. If $x=0$ and $0 \leq y \leq d-1$ then $C$ is connected to $(r-1)+y \rho^{2}$ as its -1 edge and to $(r-1)+((y-1) \bmod (d-1)) \rho^{2}$ as its $-\rho$ edge.

For example, Figure 6 illustrates the rectangular representation for EJ network generated with $\alpha=3+3 \rho$.

For simplicity, in a rectangular representation, we call the dimensions 1 , $\rho^{2}+1=\rho$, and $\rho^{2}$, in respective order, as horizontal, diagonal, and vertical dimensions.

\section{Edge Disjoint Hamiltonian Cycles when $\operatorname{gcd}(a, b)=d>1$}

From the argument in the previous section, it can be seen that there exist three sets of $d$ disjoint cycles corresponding to dimensions $1, \rho$, and $\rho^{2}$. For clarity, this is formally proved in the following theorem. 


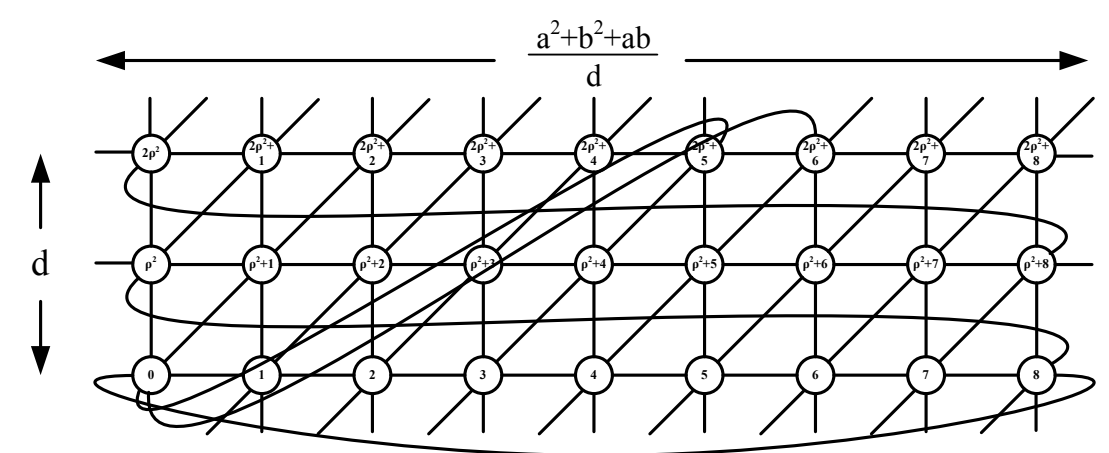

Figure 6: Rectangular Representation for EJ Network Generated with $\alpha=3+3 \rho$.

Theorem 2. Given an EJ network generated with $\alpha=a+b \rho$ where $g c d(a, b)$ $=d>1$, there exist three sets of $d$ disjoint cycles where each cycle is of length $r=\frac{N(\alpha)}{d}$. The edges in the first, second, and third sets of $d$ disjoint cycles are along, in respective order, the horizontal, the vertical, and the diagonal dimensions.

Proof. We follow a similar proof as in [1] to prove that there exists a set of $d$ disjoint cycles in the horizontal dimension. Similar proofs can be followed for the vertical and diagonal dimensions. The proof of the horizontal dimension is as follows.

Since the path has a limited number of nodes, there exists a node at distance $j, j$ is an integer $>0$, from node $\beta_{1}$ such that $\beta_{1}+j \equiv \beta_{1}(\bmod \alpha)$. i.e., we visited all the nodes in the path and returned to node $\beta_{1}$. This implies that $j \equiv 0 \bmod \alpha$. Thus, $j$ is the smallest EJ integer multiple of $\alpha$. Furthermore, we conclude that $j=\beta \alpha=(x+y \rho)(a+b \rho)=(x a-$ $y b)+(x b+(a+b) y) \rho$. Since $j$ is an integer, the coefficient of $\rho$ is zero and so $x b+(a+b) y=0$. Since $\operatorname{gcd}(a, b)=d$, we get $a=a_{1} d, b=b_{1} d$, and $\operatorname{gcd}\left(a_{1}, b_{1}\right)=1$. So, $d\left(x b_{1}+\left(a_{1}+b_{1}\right) y\right)=0$. Since $\operatorname{gcd}\left(a_{1}, b_{1}\right)=1$, we get $-\left(a_{1}+b_{1}\right) y=x b_{1}$. Thus, $y=-g b_{1}, x=g\left(a_{1}+b_{1}\right)$. Therefore, $j=(x a-y b)=\left(a g\left(a_{1}+b_{1}\right)+b g b_{1}\right)=g d\left(a_{1}^{2}+b_{1}^{2}+a_{1} b_{1}\right)=g\left(\frac{a^{2}+b^{2}+a b}{d}\right)$.

We need to prove that $g=1$. If $\frac{a^{2}+b^{2}+a b}{d} \bmod \alpha=0$, then $g=1$. Now consider

$$
\begin{aligned}
\frac{a^{2}+b^{2}+a b}{d} & =d\left(a_{1}^{2}+b_{1}^{2}+a_{1} b_{1}\right)=d\left(a_{1}+b_{1} \rho\right)\left(a_{1}+b_{1}-b_{1} \rho\right) \\
& =\left(a_{1} d+b_{1} d \rho\right)\left(a_{1}+b_{1}-b_{1} \rho\right)=(a+b \rho)\left(a_{1}+b_{1}-b_{1} \rho\right) \\
& =\alpha\left(a_{1}+b_{1}-b_{1} \rho\right)
\end{aligned}
$$


This implies $\alpha\left(a_{1}+b_{1}-b_{1} \rho\right) \bmod \alpha=0$. Thus, $g=1$. This proves, by traversing the nodes whose addresses differ by 1 , we get a cycle of length $\frac{a^{2}+b^{2}+a b}{d}$.

Furthermore, suppose $\beta_{2}$ is not in the above cycle. Then we can prove that the nodes $\beta_{2}+j, 0 \leq j<r$, form another cycle of length $r$. Now, consider a node $\beta_{3}$ which is not in the above two cycles. Then all the nodes $\beta_{3}+j, 0 \leq j<r$, form another disjoint cycle of length $r$. By continuing this process, we get $d$ disjoint cycles of length $r$. This can be proved as follows. Suppose $\gamma_{1} \equiv\left(k_{1} \rho^{2}+j_{1}\right) \bmod \alpha, 0 \leq k_{1}<d, 0 \leq j_{1}<r$ (i.e., $\gamma_{1}$ is in the $k_{1}$-th cycle). We need to prove that $\gamma_{1}$ is not in any other cycle. Suppose $\gamma_{1}$ is also equal to $\gamma_{2} \equiv\left(k_{2} \rho^{2}+j_{2}\right) \bmod \alpha, 0 \leq k_{2}<d, 0 \leq j_{2}<r$. Then $\left(k_{1} \rho^{2}+j_{1}\right) \equiv\left(k_{2} \rho^{2}+j_{2}\right) \bmod \alpha$. This implies

$$
\begin{aligned}
\rho^{2}\left(k_{1}-k_{2}\right)+\left(j_{1}-j_{2}\right) \equiv & 0 \bmod \alpha=(x+y \rho)(a+b \rho) \\
= & d\left[\left(a_{1} x-b_{1} y\right)+\rho\left(b_{1} x+y\left(a_{1}+b_{1}\right)\right)\right] \\
= & d\left[\left(a_{1} x-b_{1} y\right)+b_{1} x+y\left(a_{1}+b_{1}\right)+\right. \\
& \left.\rho^{2}\left(b_{1} x+y\left(a_{1}+b_{1}\right)\right)\right]
\end{aligned}
$$

Thus, $\left(k_{1}-k_{2}\right)=d\left[b_{1} x+y\left(a_{1}+b_{1}\right)\right]$. This implies $d \mid\left(k_{1}-k_{2}\right)$. But $\left|k_{1}-k_{2}\right|<d$ and hence $k_{1}=k_{2}$ and $j_{1}=j_{2}$. That is, we get $d$ disjoint cycles, which are of the form $\left\{i \rho^{2}+j \mid 0 \leq i<d, 0 \leq j<r\right\}$.

The horizontal (respectively, the vertical and diagonal) $d$ disjoint cycles are numbered from 0 to $d-1$ starting from the bottom to the top (respectively, left to right) of the rectangle. Figure 7, for example, illustrates the three sets of $d$ disjoint cycles in EJ network generated with $\alpha=3+3 \rho$. The graph has three node disjoint cycles in each dimension where each cycle contains 9 nodes.

Note that, as shown in Figure 7, the rectangular representation can be divided into $\frac{r}{d}$ blocks of size $d \times d$ and this idea is helpful in exchanging edges as described in the next section.

So far, we have explained that there exist three sets of $d$ node disjoint cycles in the rectangular representation. In order to get three edge disjoint Hamiltonian cycles, some edges need to be exchanged between the first and the second sets of $d$ node disjoint cycles to get the first two edge disjoint Hamiltonian cycles. In addition, we also need to do some edge exchanges between the just formed Hamiltonian cycle and the third set of $d$ cycles to form the third Hamiltonian cycle and at the same time making sure that the re-modified first one remains to be edge disjoint Hamiltonian cycle. 


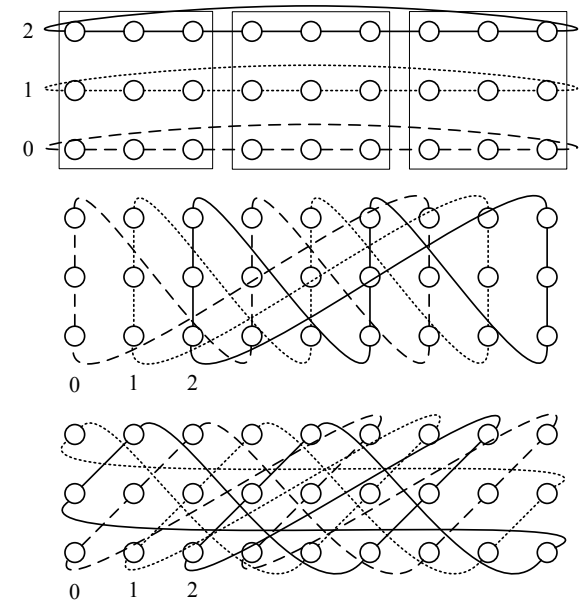

Figure 7: Three Sets of 3 Disjoint Cycles in EJ Network Generated with $\alpha=3+3 \rho$.

In order to get the first and second edge disjoint Hamiltonian cycles, we need to do some proper edge exchanges between the first (horizontal) and second (vertical) sets of $d$ node disjoint cycles in the first block of the rectangular representation.

The horizontal exchanged edges are as follows (please refer Figures 8 and 9 for an example): In the $(d-1)$-th row the first edge from left and in the 0 -th row the $(d-1)$-th edge from left. In the $i$-th row, $i=2,3, \ldots, d-2$, the $(d-i)$-th and $(d-i+1)$-th edges from left. Similarly, the vertical exchanged edges are as follows: In column $0,(d-1)$-th edge from bottom and in column $d-1$ the first edge from bottom. In the $i$-th column, $i=1,2, \ldots, d-2,(d-i)$ th and $(d-i-1)$-th edges from bottom. Table 1 formally describes these exchange edges.

By exchanging the edges descibed in Table 1, we get two edge disjoint Hamiltonian cycles as shown below. The first Hamiltonian cycle (in the horizontal dimension) can be obtained by letting $t=0$ if $d$ is odd (or $t=1$ if $d$ is even) and letting $k$ be the cycle number in the horizontal dimension. The node visiting sequence is:

1. In cycle $k=0$, starting from node 0 visit $d-2$ nodes by stepping forward along dimension 1 and then 2 steps up (using the two exchanged vertical edges).

2. For $k=2,4,6, \ldots, d-1-t$, visit all the nodes in cycles $k$ by going forward along dimension 1 except for the last node (unless $k=d-1$ 
Table 1: Proper Edge Exchanges between Horizontal and Vertical $d$ Disjoint Cycles for $i=d-2, d-3, \ldots, 1$ as Horizontal and for $j=1,2, \ldots, d-2$ as Vertical Cycles.

\begin{tabular}{|c|c|c|c|c|}
\hline Horizontal edges & Cycle \# & Exchange with & Cycle \# & Vertical edges \\
\hline$(d-1) \rho^{2},(d-1) \rho^{2}+1$ & $d-1$ & & 0 & $(d-1) \rho^{2},(d-2) \rho^{2}$ \\
\hline $\begin{array}{c}(d-2) \rho^{2} \\
(d-2) \rho^{2}+1 \\
(d-2) \rho^{2}+2\end{array}$ & $d-2$ & & 1 & $\begin{array}{l}(d-1) \rho^{2}+1 \\
(d-2) \rho^{2}+1 \\
(d-3) \rho^{2}+1\end{array}$ \\
\hline$\ldots$ & $\ldots$ & & $\ldots$ & $\ldots$ \\
\hline $\begin{array}{c}i \rho^{2}+(d-2-i), \\
i \rho^{2}+(d-2-i)+1, \\
i \rho^{2}+(d-2-i)+2\end{array}$ & $i$ & & $j$ & $\begin{array}{c}(d-j) \rho^{2}+j, \\
(d-j-1) \rho^{2}+j, \\
(d-j-2) \rho^{2}+j\end{array}$ \\
\hline$\ldots$ & $\ldots$ & & $\ldots$ & $\ldots$ \\
\hline $\begin{array}{l}\rho^{2}+(d-3), \\
\rho^{2}+(d-2), \\
\rho^{2}+(d-1)\end{array}$ & 1 & & $d-2$ & $\begin{array}{c}2 \rho^{2}+(d-2), \\
\rho^{2}+(d-2), \\
(d-2)\end{array}$ \\
\hline$(d-2),(d-1)$ & 0 & & $d-1$ & $\rho^{2}+(d-1),(d-1)$ \\
\hline
\end{tabular}

and $d$ is odd) and then go 2 steps up, i.e., use two exchanged vertical edges, (or 1 step down if $d$ is odd and $k=d-1$, or 1 step up if $d$ is even and $k=d-2$ ).

3. For $k=d-2+t, d-4+t, d-6+t, \ldots, 1$, visit all the nodes in cycles $k$ by going forward along dimension -1 , i.e., backward of dimension 1 , except for the last node (unless $k=d-1$ and $d$ is even), and then go 2 steps down, i.e., use two exchanged vertical edges, (or 1 step down if $k=1)$.

4. For $k=0$, visit all the remaining nodes in cycle $k$ until reaching node 0 .

The second Hamiltonian (in vertical dimension) cycle can be obtained by following the above algorithm but considering the vertical dimension instead. The following lemma proves that these two cycles are edge disjoint Hamiltonian cycles.

Lemma 3. Given an EJ network generated with $\alpha=a+b \rho$ with $\operatorname{gcd}(a, b)=$ d. By applying Table 1 to exchange proper edges between the horizontal and the vertical $d$ disjoint cycles, we get the first two edge disjoint Hamiltonian cycles. 
Proof. The two Hamiltonian cycles are described above. In addition, it can be seen that the $2(d-1)$ vertical edges, which are placed in the horizontal $d$ disjoint cycles to form the first Hamiltonian cycle, are not used in the vertical $d$ disjoint cycles. Similarly, the exchanged $2(d-1)$ horizontal edges are not used in the first Hamiltonian cycle. Thus, we get the first two edge disjoint Hamiltonian cycles in EJ network.

Example 3. Given an EJ generator $\alpha=9+9 \rho$ and $\operatorname{gcd}(9,9)=9$ which is odd, the first and second Hamiltonian cycles can be obtained by using Table 1 as shown in Table 2 .

Table 2: Proper Edge Exchanges between Horizontal and Vertical 9 Disjoint Cycles in EJ Network Generated with $\alpha=9+9 \rho$.

\begin{tabular}{|c|c|c|c|c|}
\hline Horizontal edges & Cycle \# & Exch. with & Cycle \# & Vertical edges \\
\hline $8 \rho^{2}, 8 \rho^{2}+1$ & 8 & & 0 & $8 \rho^{2}, 7 \rho^{2}$ \\
\hline $7 \rho^{2}, 7 \rho^{2}+1,7 \rho^{2}+2$ & 7 & & 1 & $8 \rho^{2}+1,7 \rho^{2}+1,6 \rho^{2}+1$ \\
\hline $6 \rho^{2}+1,6 \rho^{2}+2,6 \rho^{2}+3$ & 6 & & 2 & $7 \rho^{2}+2,6 \rho^{2}+2,5 \rho^{2}+2$ \\
\hline $5 \rho^{2}+2,5 \rho^{2}+3,5 \rho^{2}+4$ & 5 & & 3 & $6 \rho^{2}+3,5 \rho^{2}+3,4 \rho^{2}+3$ \\
\hline $4 \rho^{2}+3,4 \rho^{2}+4,4 \rho^{2}+5$ & 4 & & 4 & $5 \rho^{2}+4,4 \rho^{2}+4,3 \rho^{2}+4$ \\
\hline $3 \rho^{2}+4,3 \rho^{2}+5,3 \rho^{2}+6$ & 3 & & 5 & $4 \rho^{2}+5,3 \rho^{2}+5,2 \rho^{2}+5$ \\
\hline $2 \rho^{2}+5,2 \rho^{2}+6,2 \rho^{2}+7$ & 2 & & 6 & $3 \rho^{2}+6,2 \rho^{2}+6, \rho^{2}+6$ \\
\hline$\rho^{2}+6, \rho^{2}+7, \rho^{2}+8$ & 1 & & 7 & $2 \rho^{2}+7, \rho^{2}+7,7$ \\
\hline 7,8 & 0 & & 8 & $\rho^{2}+8,8$ \\
\hline
\end{tabular}

Figures 8 and 9 show the first and the second edge disjoint Hamiltonian cycles after performing the proper edge exchanges between the horizontal and vertical sets of 9 disjoint cycles.

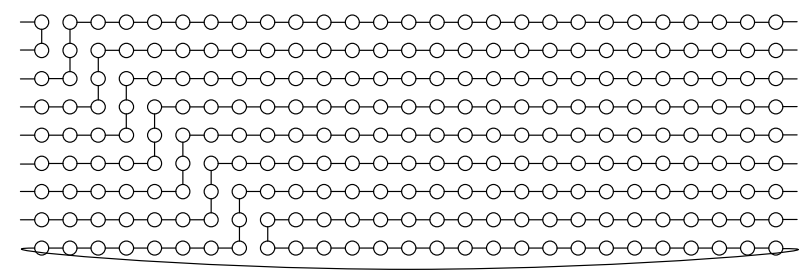

Figure 8: First Edge Disjoint Hamiltonian Cycle in EJ Network with $\alpha=9+9 \rho$ after First Edge Exchanges.

Now, to get the third edge disjoint Hamiltonian cycle, we have two cases, one in which $d$ is odd and another in which $d$ is even. When $d$ is odd, the 


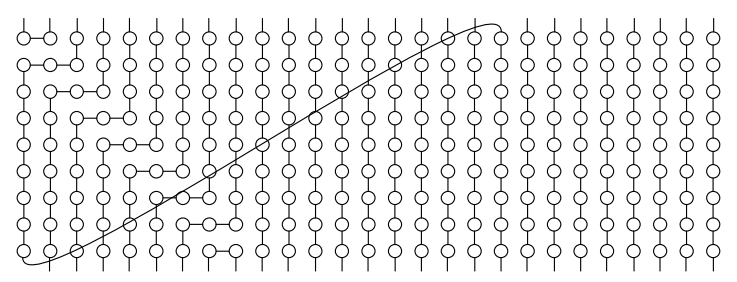

Figure 9: Second Edge Disjoint Hamiltonian Cycle in EJ Network with $\alpha=9+9 \rho$ after First Edge Exchanges.

exchanged edges are as follows (please refer Figures 10 and 11): In row 0, the $(d-1)$-th edge from right and in row $d-1$ the $(d-2)$-nd edge from right. In all other rows (i.e., rows $i, i=1,2, \ldots, d-1$ ) the edges $d-1$ and $d-2$ from right. Table 3 formally describes these edges.

Table 3: Proper Edge Exchanges between Horizontal and Diagonal $d$ Disjoint Cycles when $d$ is Odd for $i=d-2, d-3, \ldots, 1$ as Horizontal Cycles and $A=r-d$.

\begin{tabular}{|c|c|c|}
\hline Horizontal edges & Cycle \# (Horizontal) & Diagonal edges \\
\hline $\begin{array}{c}(d-1) \rho^{2}+A+1, \\
(d-1) \rho^{2}+A+2\end{array}$ & $d-1$ & $\begin{array}{c}(d-2) \rho^{2}+A, \\
(d-1) \rho^{2}+A+1\end{array}$ \\
\hline $\begin{array}{c}(d-2) \rho^{2}+A, \\
(d-2) \rho^{2}+A+1, \\
(d-2) \rho^{2}+A+2\end{array}$ & $d-2$ & $\begin{array}{c}(d-3) \rho^{2}+A, \\
(d-2) \rho^{2}+A+1, \\
(d-1) \rho^{2}+A+2\end{array}$ \\
\hline$\ldots$ & & $\ldots$ \\
\hline$i \rho^{2}+A$, & $\ldots$ & $(i-1) \rho^{2}+A$, \\
$i \rho^{2}+A+1$, & & $i \rho^{2}+A+1$, \\
$i \rho^{2}+A+2$ & $i$ & $(i+1) \rho^{2}+A+2$ \\
\hline$\ldots$ & & $\ldots$ \\
\hline$\rho^{2}+A$, & $\ldots$ & $\rho^{2}+A+1$, \\
$\rho^{2}+A+1$, & 1 & $A \rho^{2}+A+2$ \\
$\rho^{2}+A+2$ & & $A+1, \rho^{2}+A+2$ \\
\hline$A, A+1$ & 0 &
\end{tabular}

By exchanging the edges described in Table 3, we get the modified first Hamiltonian cycle and the third Hamiltonian cycle. The third Hamiltonian cycle (in the diagonal dimension) can be obtained as follows. Let $k$ be the cycle number in the diagonal dimension. The node visiting sequence is as follows: 
1. When $k=0$, starting from node 0 visit $d$ nodes by stepping along dimension $\rho^{2}+1$ (i.e., diagonal) and then 1 step forward along dimension 1. Then, we are at cycle $k=1$.

2. When $k=1$, visit all nodes in cycle $k=1$, and then go 2 steps along dimension -1 , i.e., backward of dimension 1 . Then we are at cycle $k=d-1$.

3. For $k=d-1, d-3, d-5, \ldots, 2$, visit all the nodes in the cycle $k$ except the last node (unless $k=2$; then visit all the nodes), and then go 2 steps along dimension -1 (or if $k=2$, go 1 step along dimension 1 ). Then, we are at cycle $k=3$.

4. For $k=3,5,7, \ldots, d-2$, visit all the nodes in cycle $k$ except the last node, and then go 2 steps along dimension 1 . Then we are at cycle $k=0$; go to the next step.

5. Visit all the nodes in cycle $k=0$ that are not visited in (1) until reaching node 0 .

Further, note that the edge exchanges are performed in the last block of the rectangular representation. Here, we will only explain the modification process on the first Hamiltonian cycle since it follows the same algorithm of the first Hamiltonian cycle previously described. The edge exchanges, i.e., the modifications, on the first Hamiltonian cycle are as follows:

1. The cycle $d-2$ is connected to the cycle $d-1$ through the diagonal edge $\left(L+(d-2) \rho^{2},(L+1)+(d-1) \rho^{2}\right)$.

2. For $k=d-3, d-4, \ldots, 0$, the cycle $k$ is connected to the cycle $k+2$ through the 2 diagonal edges $\left(L+k \rho^{2},(L+1)+(k+1) \rho^{2}\right)$ and $\left((L+1)+(k+1) \rho^{2},(L+2)+(k+2) \rho^{2}\right)$ passing through the node $(L+1)+(k+1) \rho^{2}$.

3. The cycle 0 is connected to the cycle 1 through the edge $((L+1),(L+$ 2) $\left.+\rho^{2}\right)$.

From the above argument, we see that the first Hamiltonian cycle, after being modified, remains a Hamiltonian cycle. Thus, we get the modified first Hamiltonian cycle and the third Hamiltonian cycle in EJ network when $d$ is 
odd. The following lemma proves that these two Hamiltonian cycles are edge disjoint.

Lemma 4. Suppose an EJ network is generated by $\alpha=a+b \rho$ with $\operatorname{gcd}(a, b)=$ $d>1$, an odd integer. As shown in Table 3, by exchanging the proper edges between the horizontal edges of the first Hamiltonian cycle and the diagonal $d$ disjoint cycles, we get two edge disjoint Hamiltonian cycles.

Proof. In the above argument, we described how to obtain the third Hamiltonian cycle when $d$ is odd by applying proper edge exchanges with the horizontal edges of the first Hamiltonian cycle, as shown in Table 3. Now, we need to prove that these two Hamiltonian cycles are edge disjoint. The proof is as follows.

First, note that the first Hamiltonian cycle and the diagonal $d$ disjoint cycles are edge disjoint since no diagonal edge is used in the first Hamiltonian cycle. In addition, it can be seen the $2(d-1)$ diagonal edges, which are placed in the first Hamiltonian cycle, are not used in the diagonal $d$ disjoint cycles. Similarly, the $2(d-1)$ horizontal edges, which are taken from the first Hamiltonian cycle, are not used in the first Hamiltonian cycle and are instead placed to the diagonal set of $d$ disjoint cycles to form a Hamiltonian cycle. Thus, these two Hamiltonian cycles are edge disjoint.

Example 4 (Continuing from Example 3). Since $\operatorname{gcd}(9,9)=9$ is odd, the third Hamiltonian cycle can be obtained by using Table 3 as shown in Table 4 .

Table 4: Proper Edge Exchanges between Horizontal and Diagonal 9 Disjoint Cycles.

\begin{tabular}{|c|c|c|}
\hline Horizontal edges & Cycle \# (Horizontal) & Diagonal edges \\
\hline $8 \rho^{2}+19,8 \rho^{2}+20$ & 8 & $7 \rho^{2}+18,8 \rho^{2}+19$ \\
\hline $7 \rho^{2}+18,7 \rho^{2}+19,7 \rho^{2}+20$ & 7 & $6 \rho^{2}+18,7 \rho^{2}+19,8 \rho^{2}+20$ \\
\hline $6 \rho^{2}+18,6 \rho^{2}+19,6 \rho^{2}+20$ & 6 & $5 \rho^{2}+18,6 \rho^{2}+19,7 \rho^{2}+20$ \\
\hline $5 \rho^{2}+18,5 \rho^{2}+19,5 \rho^{2}+20$ & 5 & $4 \rho^{2}+18,5 \rho^{2}+19,6 \rho^{2}+20$ \\
\hline $4 \rho^{2}+18,4 \rho^{2}+19,4 \rho^{2}+20$ & 4 & $3 \rho^{2}+18,4 \rho^{2}+19,5 \rho^{2}+20$ \\
\hline $3 \rho^{2}+18,3 \rho^{2}+19,3 \rho^{2}+20$ & 3 & $2 \rho^{2}+18,3 \rho^{2}+19,4 \rho^{2}+20$ \\
\hline $2 \rho^{2}+18,2 \rho^{2}+19,2 \rho^{2}+20$ & 2 & $\rho^{2}+18,2 \rho^{2}+19,3 \rho^{2}+20$ \\
\hline$\rho^{2}+18, \rho^{2}+19, \rho^{2}+20$ & 1 & $18, \rho^{2}+19,2 \rho^{2}+20$ \\
\hline 18,19 & 0 & $19, \rho^{2}+20$ \\
\hline
\end{tabular}


Figures 10 and 11 show the modified first and third edge disjoint Hamiltonian cycles after performing the proper edge exchanges between the horizontal and diagonal 9 cycles.

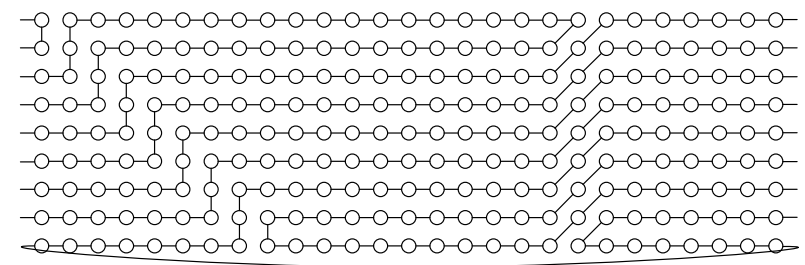

Figure 10: Modified First Edge Disjoint Hamiltonian Cycle in EJ Network with $\alpha=9+9 \rho$ after Second Edge Exchanges.

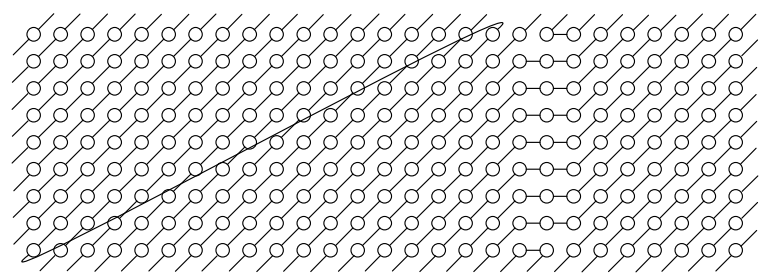

Figure 11: Third Edge Disjoint Hamiltonian Cycle in EJ Network with $\alpha=9+9 \rho$ after First Edge Exchanges.

When $d$ is even we follow Table 5 to perform some proper exchange edges between the first edge disjoint Hamiltonian cycle and the third (diagonal) set of $d$ node disjoint cycles to get the third edge disjoint Hamiltonian cycle.

The exchnage edges are as follows: In the first Hamiltonian cycle, all the even horizontal edges in the 0 -th and $(d-1)$-th rows. In the diagonal $d$ cycles, all the boundary edges are exchanged. These exchanged edges are formally defined in Table 5.

Table 5: Proper Edge Exchanges between Horizontal and Diagonal $d$ Disjoint Cycles when $d$ is Even and $A=r-d$ Based on Horizontal $d$ Disjoint Cycles for $j=0,1, \ldots, \frac{r}{2}-1$, where $r$ and $L$ are as Described in Section 4.

\begin{tabular}{|c|c|c|}
\hline Horizontal edges & Cycle \# (Horizontal) & Diagonal edges \\
\hline $\begin{array}{c}(d-1) \rho^{2}+(2 j+1 \bmod r) \\
(d-1) \rho^{2}+(2 j+2 \bmod r)\end{array}$ & $d-1$ & $\begin{array}{c}(d-1) \rho^{2}+2 j, \\
-L+2 j+1 \bmod r\end{array}$ \\
\hline $\begin{array}{c}(2 j+1 \bmod r), \\
(2 j+2 \bmod r)\end{array}$ & 0 & $\begin{array}{c}(d-1) \rho^{2}+(L+2 j-1 \bmod r), \\
j\end{array}$ \\
\hline
\end{tabular}


By exchanging the edges descibed in Table 5 on the first Hamiltonian cycle and the diagonal $d$ disjoint cycles, where $d$ is even, we get the modified first Hamiltonian cycle and the third Hamiltonian cycle. The third Hamiltonian cycle (in the diagonal dimension) can be obtained as follows. Let currentnode $=0$ be the current node during the visiting sequence. The node visiting sequence is as follows:

1. From the currentnode, we go $d-1$ steps along dimension $\rho^{2}+1$, i.e., diagonal, and then 1 step along dimension 1 . Then we are at the next cycle.

2. From the currentnode, we go $d-1$ steps along dimension $-\left(\rho^{2}+1\right)$, i.e., backward on the diagonal dimension, and then 1 step along dimension 1.

3. Repeat (1) and (2) $\frac{r}{2}$ times until we reach node 0 .

In the odd case, the edge exchanges are performed in the last block of the rectangular representation. In the even case, instead, the edge exchanges are performed in the first and last $d$ (horizontal) disjoint cycles of the rectangular representation. Below we describe the updated first edge disjoint Hamiltonian cycle after being modified.

1. Let currentnode $=0$ be the current node during the visiting sequence.

2. From the currentnode, we go 1 step along dimension 1, 1 step along dimension $-\left(\rho^{2}+1\right)$ (i.e., backward of dimension $\left.\rho^{2}+1\right)$, 1 step along dimension 1 , and then 1 step along dimension $\rho^{2}+1$.

3. We continue repeating (2) $\frac{d-2}{2}$ times until we reach node $d-2$, then we continue visiting all the nodes in the even cycles as previously described until we reach node $(d-1) \rho^{2}$; then we go 1 step along dimension $\left(\rho^{2}+1\right)$.

4. From the currentnode, we go 1 step along dimension -1 , 1 step along dimension $-\left(\rho^{2}+1\right)$, 1 step along dimension -1 , and 1 step along dimension $\left(\rho^{2}+1\right)$.

5. Repeat (4) $\frac{(r-L+1)-(d-1)}{2}$ times until we reach node $d-1$. Then we continue visiting all the nodes in the odd cycles, as previously described, until we reach node $(d-1) \rho^{2}+1$. Then, we go 1 step along dimension $\left(\rho^{2}+1\right)$. 
6. From the currentnode, we go 1 step along dimension 1, 1 step along dimension $-\left(\rho^{2}+1\right)$, 1 step along dimension 1 , and 1 step along dimension $\left(\rho^{2}+1\right)$.

7. Repeat (6) $\frac{L-2}{2}$ times until we reach node 0 .

From the above argument, we see that the first Hamiltonian cycle, after being modified, remains a Hamiltonian cycle. Thus, we get the modified first Hamiltonian cycle and the third Hamiltonian cycle in EJ network when $d$ is even. The following lemma proves that these two Hamiltonian cycles are edge disjoint.

Lemma 5. Suppose an EJ network is generated by $\alpha=a+b \rho$ with $\operatorname{gcd}(a, b)=$ $d>1$, an even integer. As shown in Table 5, by exchanging the proper edges between the horizontal edges of the first Hamiltonian cycle and the diagonal $d$ disjoint cycles, we get two edge disjoint Hamiltonian cycles.

Proof. In the above argument, we described how to obtain the third Hamiltonian cycle when $d$ is even by applying proper edge exchanges with the horizontal edges of the first Hamiltonian cycle, as shown in Table 5. Now, we need to prove that these two Hamiltonian cycles are edge disjoint. The proof is as follows.

It can be seen that the $r$ diagonal edges, which are placed in the first Hamiltonian cycle, are not used in the diagonal $d$ disjoint cycles. Similarly, the $r$ horizontal edges, which are taken from the first Hamiltonian cycle and placed to the diagonal set of $d$ disjoint cycles to form a Hamiltonian cycle are not used in the first Hamiltonian cycle. Thus, the two Hamiltonian cycles are edge disjoint.

The following theorem summaries the main results.

Theorem 3. Given an EJ network generated with $\alpha=a+b \rho$ and $\operatorname{gcd}(a, b)=$ $d>1$, there exist three edge disjoint Hamiltonian cycles.

Proof. In Lemma 3, we proved that the first two edge disjoint Hamiltonian cycles could be generated by performing proper edge exchanges, as shown in Table 1, between the horizontal and the vertical sets of $d$ disjoint cycles. In order to find the third edge disjoint Hamiltonian cycle, we divided the problem into two cases, when $d$ is odd and when it is even. 
In Lemma 4, when $d$ is odd, we proved that the third edge disjoint Hamiltonian cycle could be obtained by performing some edge exchanges, as shown in Table 3, between the horizontal edges of the first Hamiltonian cycle and the edges of the diagonal set of $d$ disjoint cycles while the first Hamiltonian cycle remains Hamiltonian cycle. Now we need to prove that the first Hamiltonian cycle, after being modified, is also an edge disjoint Hamiltonian cycle with the second Hamiltonian cycle. In addition, we need to prove that the second Hamiltonian cycle is also edge disjoint with the third Hamiltonian cycle. The proof is as follows.

The first Hamiltonian cycle is based on the horizontal set of $d$ disjoint cycles and contains $2(d-1)$ vertical edges that are not in the second and third Hamiltonian cycles. In addition, it also contains $2(d-1)$ diagonal edges that are not in the second and third Hamiltonian cycles. Thus, the first Hamiltonian cycle is edge disjoint with the second and third Hamiltonian cycles. Further, the second Hamiltonian cycle is based on the vertical set of $d$ disjoint cycles and contains $2(d-1)$ horizontal edges that are not used in the first and third Hamiltonian cycles, i.e., no common edges. Thus, the second Hamiltonian cycle is edge disjoint with the third Hamiltonian cycle. Since each Hamiltonian cycle is edge disjoint with the others we get three edge disjoint Hamiltonian cycles in EJ network when $d$ is odd.

Similarly, when $d$ is even, the proof is same as above except use Lemma 5 (instead of Lemma 4). That is, the first Hamiltonian cycle is based on the horizontal set of $d$ disjoint cycles and contains $2(d-1)$ vertical edges that are not in the second and third Hamiltonian cycles. In addition, it also uses $r$ diagonal edges that are not used in the second and third Hamiltonian cycles. Thus, the first Hamiltonian cycle is edge disjoint with the second and third Hamiltonian cycles. Further, the second Hamiltonian cycle is based on the vertical set of $d$ disjoint cycles and contains $2(d-1)$ horizontal edges that are not used in the first and third Hamiltonian cycles, i.e., no common edges. Thus, the second Hamiltonian cycle is edge disjoint with the third Hamiltonian cycle. Since each Hamiltonian cycle is edge disjoint with the others we get three edge disjoint Hamiltonian cycles in EJ network when $d$ is even.

Figures 9, 10, and 11 show three edge disjoint Hamiltonian cycles in the EJ network generated with $\alpha=9+9 \rho$. 


\section{Conclusion}

In parallel systems, many communication algorithms can be efficiently solved by finding edge disjoint Hamiltonian cycles in the underlying interconnection network. This paper discusses the optimal methods for finding 3 edge disjoint Hamiltonian cycles in an EJ network generated by $\alpha=a+b \rho$, when $\operatorname{gcd}(a, b) \geq 1$, by using the rectangular representation of the network. The methods proposed in this paper form the basis for finding edge disjoint Hamiltonian cycles and edge disjoint toroidal networks in multi-dimensional EJ networks and these results will be presented in a future paper.

\section{Acknouwledgement}

This work is supported by the NSF Grant CCF-1015804. The authors also extend their appreciation to the Deanship of Scientific Research at King Saud University for funding this work through research group no. RGP-264.

\section{References}

[1] Albader, B., 2009. Some communication algorithms for Gaussian and Eisenstein-Jacobi networks. Ph.D. thesis, Oregon State University, Corvallis, OR, USA, aAI3376768.

[2] Albader, B., Bose, B., Flahive, M., January 2012. Efficient communication algorithms in hexagonal mesh interconnection networks. IEEE Trans. Parallel Distrib. Syst. 23 (1), 69-77.

[3] Anantha, M., Bose, B., AlBdaiwi, B. F., October 2007. Mixed-radix Gray codes in Lee metric. IEEE Transactions on Computers 56 (10), 1297-1307.

[4] Bae, M., Albdaiwi, B. F., Bose, B., 2004. Edge-disjoint Hamiltonian cycles in two-dimensional torus. International Journal of Mathematics and Mathematical Sciences 2004 (25), 1299-1308.

[5] Bae, M., Bose, B., 2000. Gray codes for torus and edge disjoint Hamiltonian cycles. In: Proceedings. 14th International Parallel and Distributed Processing Symposium. pp. 365-370. 
[6] Bae, M., Bose, B., October 2003. Edge disjoint Hamiltonian cycles in kary n-cubes and hypercubes. IEEE Transactions on Computers 52 (10), $1271-1284$.

[7] Bertekas, D., Tsitskilis, J., 1989. Parallel and Distributed Computation: Numerical Methods. Prentice-Hall.

[8] Duato, J., Yalamanchili, S., Ni, L., 1997. Interconnection Networks: An Engineering Approach, 1st Edition. IEEE Computer Society Press, Los Alamitos, CA, USA.

[9] Flahive, M., Bose, B., August 2010. The topology of Gaussian and Eisenstein-Jacobi interconnection networks. IEEE Transactions on Parallel and Distributed Systems 21 (8), 1132-1142.

[10] Grama, A., Gupta, A., Karypis, G., Kumar, V., 2003. Introduction to Parallel Computing, 2nd Edition. Addison-Wesley Longman Publishing Co., Inc., Boston, MA, USA.

[11] Hardy, G. H., Wright, E. M., April 1980. An Introduction to the Theory of Numbers (Oxford Science Publications), 5th Edition. Oxford University Press, USA.

[12] Jha, P., Prasad, R., Aug 2012. Hamiltonian decomposition of the rectangular twisted torus. Parallel and Distributed Systems, IEEE Transactions on 23 (8), 1504-1507.

[13] Latifi, S., Zheng, S.-Q., April 1993. On link-disjoint Hamiltonian cycles of torus networks. In: Proceedings. IEEE Southeastcon '93. p. 5 p.

[14] Martinez, C., Beivide, R., Stafford, E., Moreto, M., Gabidulin, E., August 2008. Modeling toroidal networks with the Gaussian integers. IEEE Transactions on Computers 57 (8), 1046-1056.

[15] Martínez, C., Stafford, E., Beivide, R., Gabidulin, E. M., March 2008. Modeling hexagonal constellations with Eisenstein-Jacobi graphs. Probl. Inf. Transm. 44, 1-11.

URL http://portal .acm.org/citation. cfm?id=1375406.1375412

[16] Rowley, R., Bose, B., April 1991. Edge-disjoint Hamiltonian cycles in De Bruijn networks. In: Proceedings. The Sixth Distributed Memory Computing Conference. pp. 707-709. 
[17] Rowley, R., Bose, B., December 1993. On the number of arc-disjoint Hamiltonian circuits in the De Bruijn graphs. Parallel Processing Letters 3 (4), 375-382. 